\title{
Behavioral phenotyping of a murine model of Alzheimer's disease in a seminaturalistic environment using RFID tracking
}

\author{
Lars Lewejohann, Anne Marie Hoppmann, Philipp Kegel, \\ Mareike Kritzler, Antonio Krüger, ANd Norbert SAChSer \\ University of Münster, Münster, Germany
}

\begin{abstract}
Neurodegenerative disorders such as Alzheimer's disease (AD) are increasingly threatening public health. Most animal models of AD consist of transgenic mice that are usually housed singly or in unisexual groups in small barren cages. Such restricted environments, however, prevent the mice from showing a variety of speciesspecific behaviors and consequently may constrain comprehensive behavioral phenotyping. On the other hand, allowing the animals to freely organize their lives in a spacious physically and socially enriched environment makes behavioral phenotyping laborious and time consuming. Radio frequency identification (RFID) using a network of antennae and small glass-coated transponders labeling each individual allows for gathering spatiotemporal information about a large number of individuals in parallel. The aim of this project was to use the RFID technique to facilitate the characterization of mice carrying a genetic disposition to develop AD-like pathology and of their wild-type conspecifics in a spacious seminaturalistic environment.
\end{abstract}

Alzheimer's disease (AD), characterized by a progressive cognitive decline, typically is accompanied by a variety of noncognitive behavioral symptoms, such as aggressiveness, wandering, agitation, restlessness, or rhythm disturbances; these traits are often referred to as "behavioral and psychological symptoms of dementia" (Lanari, Amenta, Silvestrelli, Tomassoni, \& Parnetti, 2006; Marshall, Fairbanks, Tekin, Vinters, \& Cummings, 2006). Obviously, such symptoms severely affect the dayto-day lives of $\mathrm{AD}$ patients, their relatives, and nursing staff. Yet, in murine models of $\mathrm{AD}$, a vast majority of the behavioral investigations have focused solely on assessing learning and memory performance, whereas knowledge about the effects of AD symptoms on the day-to-day lives of the mice, especially with regard to social behavior in the broadest sense, is scarce.

Manually assessing the spontaneous behavior of mice is laborious and time consuming. To circumvent such constraints, some approaches have already been made to automate assessment of the home cage behavior of mice (de Visser, van den Bos, Kuurman, Kas, \& Spruijt, 2006; Galsworthy et al., 2005). However, these systems operate on a relatively small scale, imposing limits in the number of animals and the complexity of their social relations.

Human as well as murine societies are characterized by social stratification; that is, resources are distributed unequally between subjects of differing social statuses. As a result, a strong association between social status and health is generally accepted (Sapolsky, 2005; von Holst,
1998). However, to our knowledge, this has not so far been proven in animal models of neurodegenerative diseases. For this reason, our study initially aimed at elucidating the effects of social status on behavior and neuropathology in a model of AD in mice housed in a spacious indoor enclosure. Therefore, we bred a mixed population of transgenic and wild-type mice in a seminaturalistic environment (SNE) and applied an automated radio frequency identification (RFID) tracking system that we developed for this purpose to monitor the mice on a 24-h/7-day basis using a network of antennae placed at strategically chosen spots in the SNE. The data derived from this system were validated through manual observations. We report here on the application and evaluation of the RFID tracking system in the SNE and discuss the usability of this system for automatically analyzing the behavior of (transgenic) mouse populations.

\section{Method}

Animals. The mice studied in this investigation were wild-type and transgenic CRND8 mice (Janus et al., 2000), which carry a double-mutated ("Swedish" and "Indiana"; K670N/M671L, V717F) form of the human amyloid precursor protein 695 (APP). Transgenic animals of this line are characterized by amyloid deposition and cognitive impairments already present at an age of 3 months (Chishti et al., 2001). One hemizygous $(+/-)$ transgenic male mouse and 3 wild-type $(-/-)$ female mice were introduced into a seminaturalistic enclosure and could reproduce freely. All

L. Lewejohann, ljohann@phenotyping.com 
3 females became pregnant and gave birth to pups of both genotypes. The population was allowed to grow up to a size of 40 adults. Tissue samples were taken from the tail tips of the mice at an age of 27 days to identify genotypes by polymerase chain reaction amplification of a DNA fragment within the promoter region of the transgene (Chishti et al., 2001). Mice were individually marked using subcutaneously injected RFID transponders as well as a color-coding scheme on their tails and ears.

Seminaturalistic enclosure. The SNE (Figure 1A) was realized as a wired indoor cage measuring $1.75 \times$ $1.75 \mathrm{~m}$ with a height of $2.10 \mathrm{~m}$. The ground floor was divided into two partitions connected by a Plexiglas tube. In addition, three wooden floors measuring $0.5 \mathrm{~m}^{2}$ each were installed in the SNE building, creating an effective surface area of about $4.5 \mathrm{~m}^{2}$. The floors contained sawdust bedding that could easily be changed when soiled. Food (Altromin 1324, Altromin $\mathrm{GmbH}$ ) and tap water were available ad lib on each floor. The mice could emigrate from the SNE by passing through a Plexiglas tube, swimming through a water basin, and finally entering an emigration cage in which they could be picked up by the experimenter (Figure 1B). A total of 29 antennae were installed in the SNE to monitor positional data of the individual mice. Connections between different floors and partitions, as well as the entrance to the emigration cage, were constructed from Plexiglas tubes with an antenna at each end to determine the direction a mouse was moving through the tube. On each floor was an additional tube with two antennae monitoring the activity in each partition. In addition, all water bottles were equipped with antennae to monitor drinking behavior. Finally, a balance that could be entered by the mice through two antenna-monitored tubes was placed in the left partition of the ground floor for automatically obtaining weight data for individual mice.

Radio frequency identification. The RFID system (Trovan Electronic Identification Systems) consisted of readers (LID 665 Miniature OEM Boards), ring antennae (air-core coil antennae for the LID 665), and animal glass transponders (ID 100). All mice carried a passive integrated transponder (PIT) injected subcutaneously into the neck region using a specially designed injector (IID 100). Each PIT had a unique ID comprising a 10-digit hexadecimal number. The ID was read while a mouse traversed the electromagnetic field established by the ring antennae-for example, when passing through tubes or visiting drinking places. The minimum distance between two antennae was $20 \mathrm{~cm}$. The readers were able to read several transponders at a maximum rate of $26 \mathrm{~Hz}$.

Configuration of the RFID readers and storage of the data in a relational database were realized by in-housedeveloped "JerryTS2" software written especially for this purpose. The software was written using the Java programming language and will be made available to researchers on demand.

Each time an antenna made contact with a transponder, a data set was created that consisted of the data set ID, date, hours, minutes, seconds, milliseconds, antenna ID, and transponder ID. These data were stored online in a re- lational database (Kritzler, Lewejohann, Krüger, Raubal, \& Sachser, 2006). Basically, this setup allowed for constant monitoring of several locomotion, activity, and behavioral patterns on a 24-h/7-day basis.

Manual observations. To validate the automated RFID tracking system, direct observation of movement data was

\section{A}

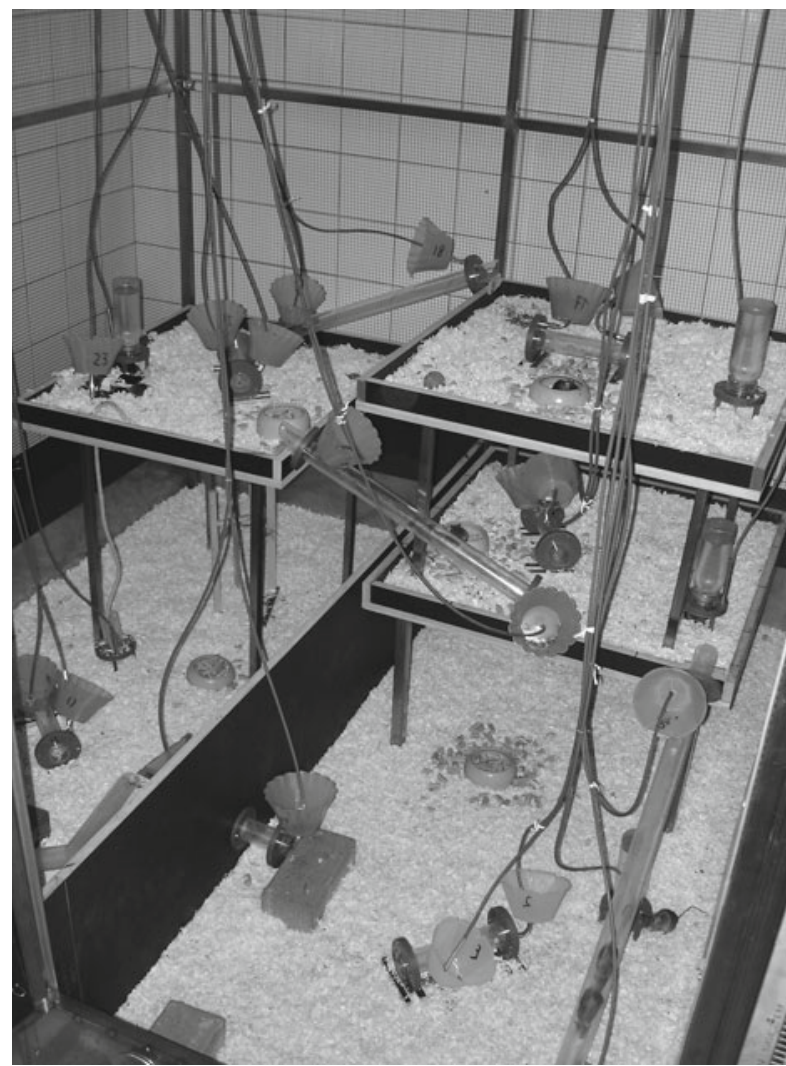

B

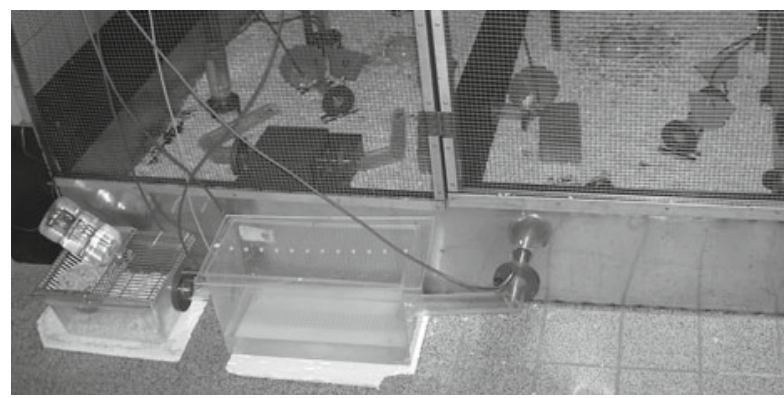

Figure 1. (A) Semi-naturalistic enclosure (SNE). Up to 40 individually RFID-labeled adult mice used the spacious indoor enclosure with an effective surface area of $4.5 \mathrm{~m}^{2}$. The SNE was equipped with 29 ring antennae reading the transponder code whenever a mouse passed through it. (B) Adjacent to the SNE, an emigration cage was placed. Mice could leave the SNE, swim across a water basin and enter the emigration cage. Two ring antennae monitored whenever an emigration event took place. 
conducted. In brief, the tracks of randomly chosen focal animals were sketched on data sheets printed with a plan of the levels of the enclosure in red ink. These data sheets were then scanned into digital images. The red lines of the SNE plan were removed, and the remaining lines representing the tracks of the mice were measured using an inhouse-written macro for Optimas image analysis software (Dunn, 1998). The number of antenna contacts triggered within one time frame was retrieved from the database, and a correlation analysis with the measured path length was performed.

Social stratification was manually analyzed by counting agonistic interactions. The spontaneous behavior of male mice was observed during their activity phase during the light period four to five times per week for 7 months. Data on agonistic behavior were recorded using behavior sampling and continuous recording (Martin \& Bateson, 1993) according to the behavioral patterns listed in Table 1 .

Elo rating. Albers and de Vries (2001) have suggested using Elo rating (developed to rate human chess players) to assess the dominance hierarchies in animal societies. In brief, this method estimates the rank of individuals over relatively long periods of time by taking into account different propensities to win or lose on the basis of the strength of an individual, known from the outcomes of previous interactions. A major advantage of this technique is that it allows for the rapid monitoring of changes in the dominance structure of a population. Agonistic interactions were noted, including the date, winner, loser, behavioral pattern, and location at which the interaction took place. These data were saved in a tab-separated text file with one interaction per row. An in-house-developed software application (ELO-Rating, also available on demand) was used to calculate tables of Elo points representing the course of dominance relationships for different territories within the SNE. All mice started with 1,000 Elo points. With each agonistic encounter, the winner gained Elo points while the loser lost them. Individuals with high Elo values were considered as being likely to win an interaction and gained only small numbers of Elo points if they won as expected. If they lost against the odds, however, their Elo value decreased substantially. For a detailed description of the procedure, see the description by Albers and de Vries.

Barnes maze. Learning and memory performance was assessed using a Barnes (1979) maze. It was considered impossible to conduct this test outside the SNE in an adjacent Barnes maze because mice raised in seminaturalistic conditions are likely to flee from such an apparatus more readily than mice raised in standard housing conditions. Therefore, a Barnes maze was built within the SNE. The apparatus consisted of a circular platform with a diameter of $70 \mathrm{~cm}$ placed $50 \mathrm{~cm}$ above the ground. At the edge of the platform were 12 holes of $3-\mathrm{cm}$ diameter, placed at equal distances. All but one of the holes led into closed tubes, $4.5 \mathrm{~cm}$ deep, made of wire mesh. The remaining hole was connected to a passageway allowing for escape from the platform to the ground level of the SNE. A trial was preceded by placing a test subject within a plastic cylinder at the center of the Barnes maze for $1 \mathrm{~min}$. To start a trial, the cylinder was lifted from outside the SNE by pulling on an attached cord. After each run, the platform was cleaned with $70 \%$ ethanol to eliminate olfactory clues. Two trials per day were held on 5 consecutive days with 3 transgenic and 15 wild-type male mice at about 147 days of age. The learning performance of both genotypes was analyzed by measuring the area under the curve in the acquisition phase from Trials 2 to 8 . During the last 2 trials, the position of the hole leading from the Barnes maze was changed in order to analyze whether the mice used spatial cues or a random strategy to escape from the platform. As measures of learning success, the number of errors (exploration of holes that could not be used to escape the platform) and escape latency were recorded.

Statistics. Graphs were created and statistics calculated using the R software package (R Development Core Team, 2008). Pearson's product-moment correlation was calculated for a correlational analysis of manually tracked path length and antenna contacts. Survival analysis was carried out using log-rank statistics. The survival rates of males and females were compared using Fisher's exact test. The number of antenna contacts triggered by mice characterized as being either dominant or subdominant were compared using unpaired two-sample Wilcoxon tests. Learning and memory performance was analyzed via an ANOVA on the areas under the learning curves (Trials 2-8), with genotype as the between-subjects factor. For all data, a significance level of $\alpha \leq .05$ was chosen.

\section{Results and Discussion}

A total of 55 mice, 21 transgenic and 34 wild-type, were raised during the course of the investigation, which lasted for 260 days in total. Of these 55 mice, 35 lived in the enclosure until the age of 150 days. During the course of the investigation, 7 males (6 transgenic and 1 wild-type) and 8 females (4 transgenic and 4 wild-type)

Table 1

Ethogram of the Behavioral Patterns Used for Analyzing Dominance Relationships

\begin{tabular}{ll}
\hline \multicolumn{1}{c}{ Behavior } & \multicolumn{1}{c}{ Description } \\
\hline Bite & A mouse drives its mouth against the fur, the skin, or the tail of another mouse. \\
Approach & A mouse moves toward a conspecific until both are within a maximum distance of one body length. \\
Attack & A mouse approaches or lunges at another mouse and then bites it. \\
Fight & Two mice struggle with each other with physical contact. Other agonistic behavior patterns are usually included. \\
Chase & A mouse runs after another mouse. \\
Follow with biting & A mouse bites the tail, caudal fur, or testicles of another mouse while both are moving. \\
Retreat & A mouse approaching or being approached moves straight away from the conspecific without being followed. \\
Flee & A mouse moves rapidly away from another mouse for a distance of at least two body lengths. \\
\hline
\end{tabular}


died. A total of 4 subdominant males ( 2 transgenic and 2 wild-type) and 1 wild-type female emigrated from the SNE. These animals were between 6 and 15 weeks of age, a time at which emigration in feral house mice has been previously described (Gerlach, 1996; Nelson, Johnson, Matter, \& Mannan, 2002). The 35 mice that reached an age of 150 days comprised 11 wild-type and 6 transgenic females and 15 wild-type but only 3 transgenic males. After 32 weeks, the transgene had completely vanished from the population because of a higher mortality rate for the transgenics than for the wild types (exact log-rank test, $Z=4.52, p<.001)$. However, this was not unexpected, given the results from previous publications (Chishti et al., 2001; Sebastiani et al., 2006) and our own observations (Lewejohann et al., 2009). The higher mortality for transgenic males than for transgenic females was not significant (Fisher's exact test for count data, $p>.28$ ). The path lengths covered by 31 different mice were assessed by manually tracking subjects of both genotypes and sexes during phases of locomotion activity. The total number of antenna contacts triggered at the same time correlated positively with the manually measured path length (Pearson's product-moment correlation, $r=.744, p<.001$ ), indicating that automated RFID tracking results reasonably reflect locomotion activity.

Agonistic behavior among the wild-type and transgenic males was assessed by manually observing offensive ag- gressive behavior (attack, fight, follow with biting) as well as avoidance behavior (be attacked, retreat, flee). In total, over 3,300 observed agonistic interactions were used to analyze the dominance relationships. Initially, the founder male dominated all other males, but he was defeated after about 5 weeks and died shortly afterward. Younger males took over parts of the enclosure, and finally the SNE was divided into four separate territories held by different dominant males. Whenever a former territory owner died or was taken out of the SNE, other males took over immediately. Elo rating revealed despotic dominance relationships, with 1 male dominating several other males, who in turn did not diverge much from each other in their ratings. When territory holders entered the territories of other males, they were subdominant to the holder of that territory and showed patterns of avoidance behavior. For this reason, separate Elo ratings were calculated for each territory, rather than calculating one overall rating that would have included all males. A sample Elo rating calculated for the left side of the ground level of the SNE is depicted in Figure 2.

Of the 3 surviving transgenic males, 1 was characterized as being dominant, indicating that the APP transgene did not restrict a mouse from becoming a territory owner.

Dominant males patrol their territory borders more frequently than subdominants, and our dominant males thereby triggered significantly more antenna contacts

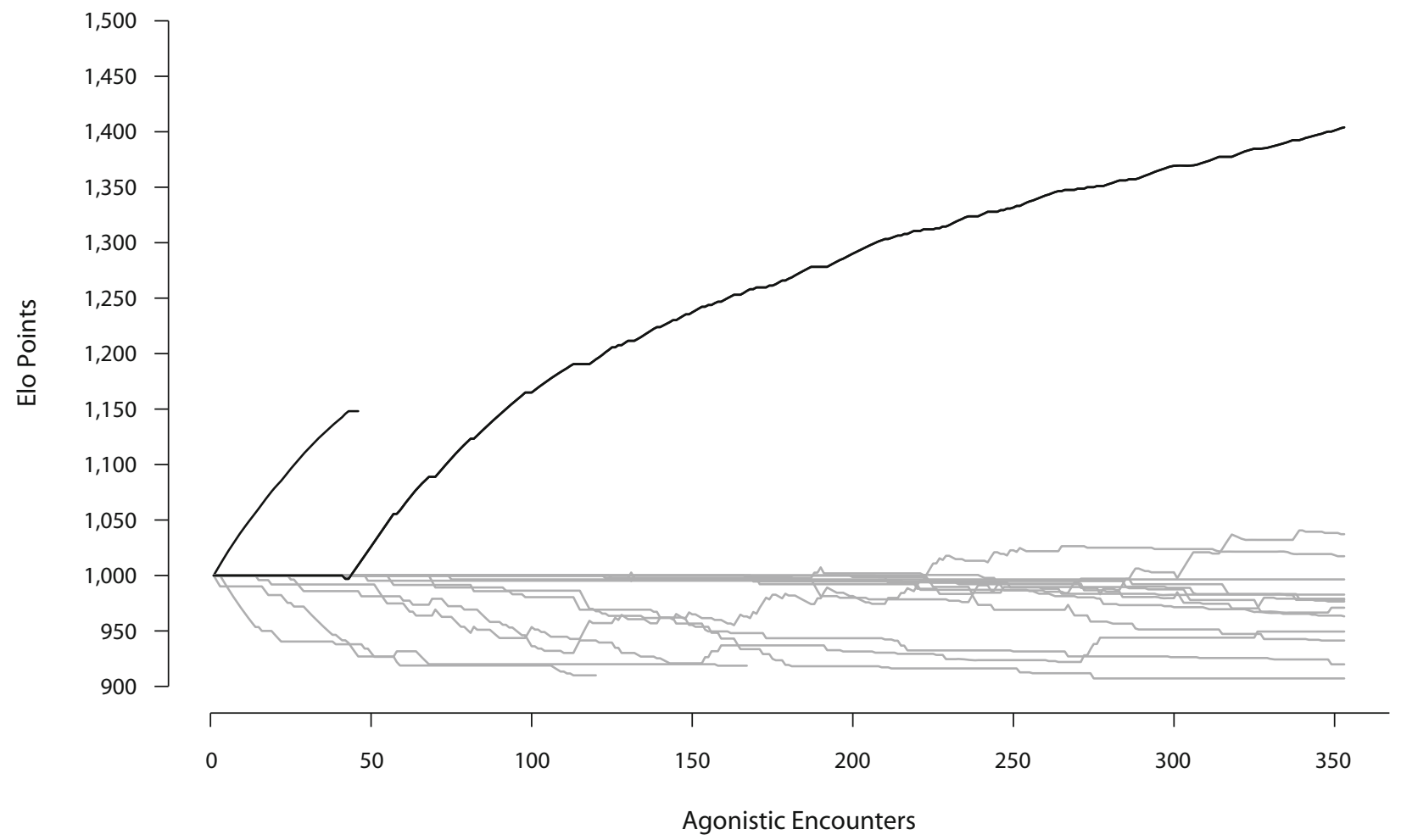

Figure 2. Elo rating, originally from chess ranking, is a tool that provides sequential estimation of dominance strength on the basis of the actual sequence of dominance interactions rather than on total wins and defeats. The figure shows the Elo points of 2 dominant males (black) and 15 subdominant males (gray) observed on the left side of the ground level in the SNE. In the course of agonistic interactions, winners gained points while the losers' points decreased. After the first dominant male died, the dominant position was taken over by another male. 


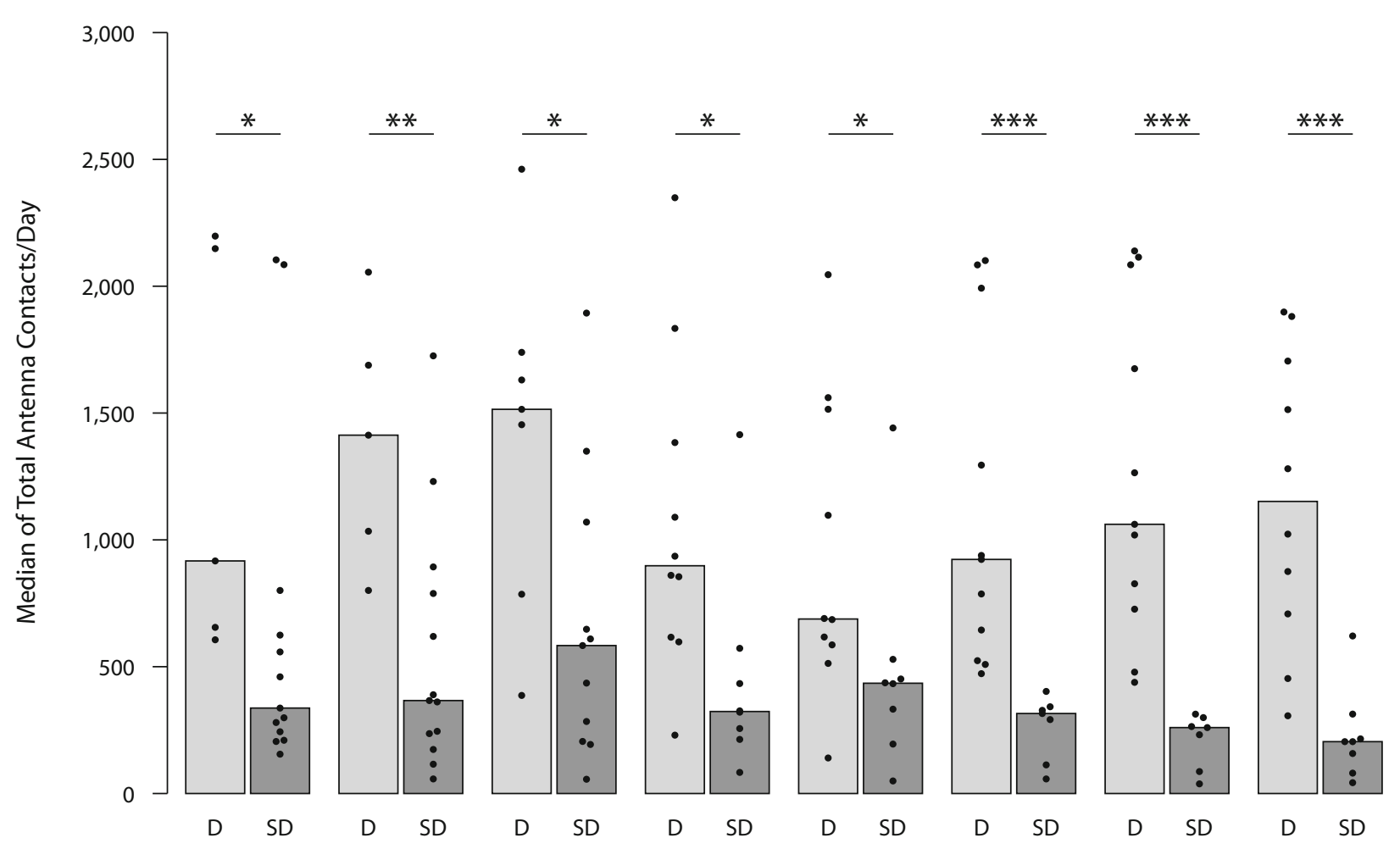

Day 70-79 Day 80-89

Day 90-99 Day 100-109

Day 110-119 Day 120-129

Day 130-139

Day 140-149

Figure 3. Antenna contacts of dominant (D) and subdominant (SD) male mice at different ages. Individual values (dots) and the median number of antenna contacts per day are shown for 10-day intervals. Dominant males triggered significantly more antenna contacts than did subdominant males. Statistics are from unpaired two-sample Wilcoxon tests. ${ }^{*} p<.05 .{ }^{* *} p<.01 .{ }^{* * *} p<.001$.

than did subdominant animals (Figure 3). This is in line with previous observations of mice, which have described higher activity in dominant than in subdominant animals (Lloyd, 1975; Mackintosh, 1970). Moreover, this result indicates that the RFID system applied here may indeed facilitate behavioral observations, because it allows for determining the social status of a male by its number of antenna contacts. With the knowledge gained in this investigation of where to place antennae within the SNE, an automated system to distinguish between dominant and subdominant males seems to be at hand.

The RFID technique has been used before in naturalistic setups (Lewejohann et al., 2004), but the density of the antenna network in that study was much lower and an in-depth evaluation of the system via manual observations was not performed.

To test spatial memory performance, a modified Barnes maze test was conducted in the SNE. Transgenic males performed significantly worse than wild types $[F(1,16)=$ $21.44, p<.001$; see Figure 4]. Thus, this test revealed that the learning and memory deficits previously recorded for TgCRND8 mice are still present in the SNE.

In this setup, the mice had the opportunity to emigrate from unfavorable conditions by swimming through a water basin into an emigration cage placed adjacent to the SNE. In total, only a few mice emigrated from the SNE. Unfor- tunately, 2 transgenic males left the SNE by emigration, reducing the total number of transgenic males in that environment to 3 . For this reason and because of the higher mortality of transgenic mice, the initially planned analysis of the relation between dominance status and AD could not be accomplished using this setup. Nevertheless, the founder male and another male born in the SNE carrying the mutated APP transgene were characterized as dominant. This confirms previous observations that the day-today life of AD mice is not compromised when the genetic predisposition interacts with a generous physically and socially enriched environment (Lewejohann et al., 2009). Most interestingly, learning deficits in a spatial memory task known from this mouse model (Görtz et al., 2008; Janus et al., 2000) persist in the SNE, although we admit the low sample size of transgenics that could be tested.

Transgenic mice did not differ from the wild types with regard to numbers of antenna contacts, indicating that daily activity was not affected by genotype. This result contrasts with previous observations from our own lab showing that transgenic CRND8 mice were more active and showed more stereotypic behavior when they were housed in standard-sized cages (Ambrée et al., 2006; Touma et al., 2004). In line with another recent study conducted in an SNE (Lewejohann et al., 2009), no stereotypic behavior was observed at all within the study period. 


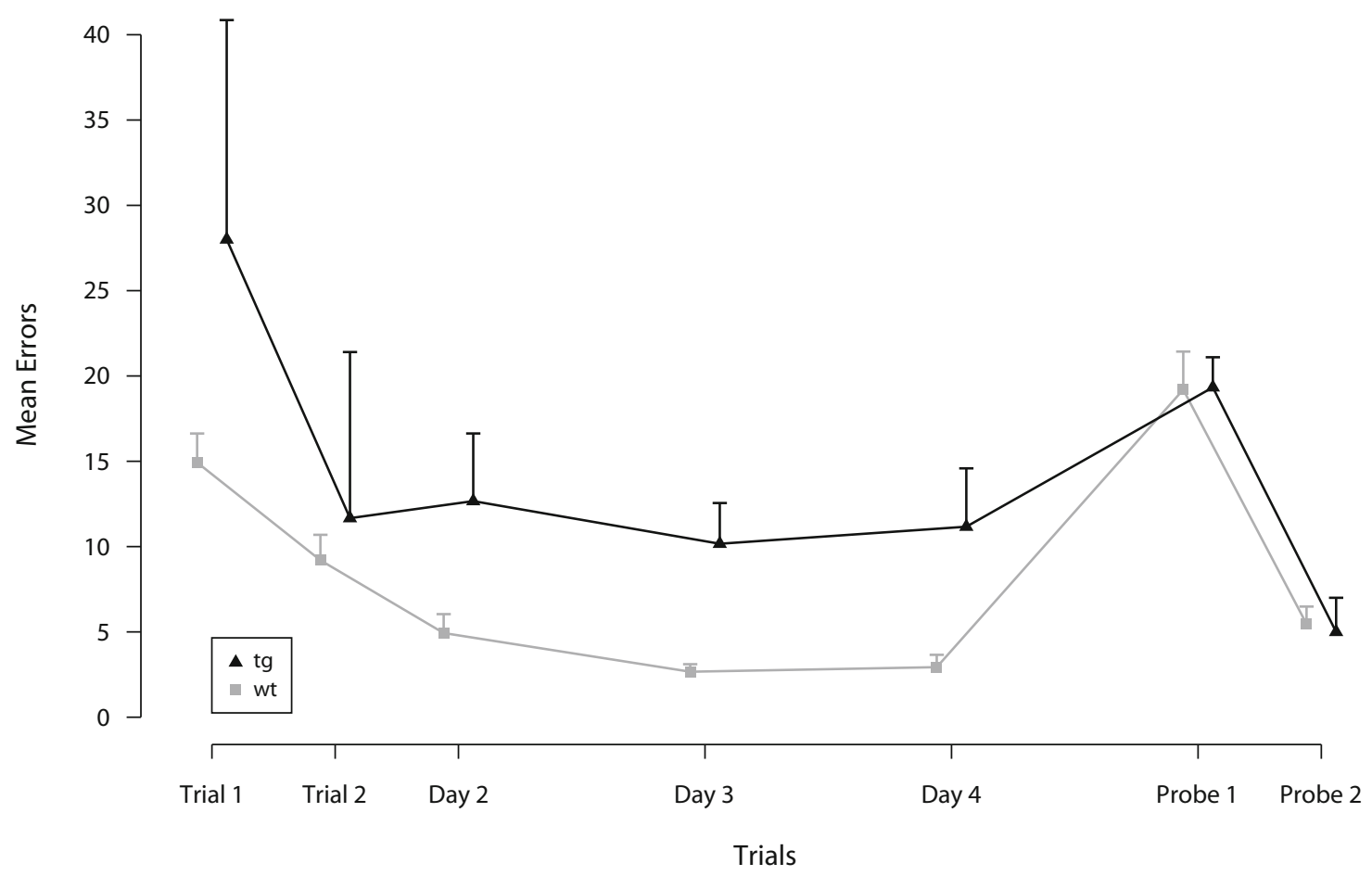

Figure 4. Spatial memory performance of transgenic and wild-type male mice on a Barnes maze. Numbers of errors are shown as mean $+S E M$. ANOVA revealed a significant effect of genotype, with transgenic males performing worse than wild types in the acquisition phase from Day $1 /$ Trial 2 to Day 4/Trial 8.

\section{Conclusion}

The RFID system installed in the SNE allowed for automatically tracking large numbers of animals at the same time. Validation of the automatically derived data by manually tracking individual mice revealed that the number of antenna contacts indeed gives a very good estimate of locomotion behavior. We extended the usability of the system by analyzing the social dominance relationships of the male mice and correlated these data with RFID-tracked activity. This setup allows researchers to exploit the full potential of comprehensive behavioral phenotyping of transgenic mice in a complex environment that typifies the environment in which the mouse genome evolved.

\section{AUTHOR NOTE}

Correspondence related to this article may be sent to L. Lewejohann, Dept. of Behavioral Biology, University of Münster, Badestr. 13, 48149 Münster, Germany (e-mail: ljohann@phenotyping.com).

\section{REFERENCES}

Albers, P. C. H., \& DE VRIes, H. (2001). Elo-rating as a tool in the sequential estimation of dominance strengths. Animal Behaviour, 61, 489-495. doi:10.1006/anbe.2000.1571

Ambrée, O., Touma, C., Görtz, N., Keyvani, K., Paulus, W., Palme, R., \& SAChSER, N. (2006). Activity changes and marked stereotypic behavior precede $\mathrm{A}^{\beta}$ pathology in TgCRND8 Alzheimer mice. Neurobiology of Aging, 27, 955-965. doi:10.1016/ j.neurobiolaging.2005.05.009

BARNES, C. A. (1979). Memory deficits associated with senescence: A neurophysiological and behavioral study in the rat. Journal of Comparative \& Physiological Psychology, 93, 74-104.

Chishti, M. A., Yang, D.-S., Janus, C., Phinney, A. L., Horne, P., PeArson, J., ET AL. (2001). Early-onset amyloid deposition and cog- nitive deficits in transgenic mice expressing a double mutant form of amyloid precursor protein 695. Journal of Biological Chemistry, 276, 21562-21570. doi:10.1074/jbc.M100710200

de Visser, L., van den Bos, R., Kuurman, W. W., Kas, M. J. H., \& SPRUIJT, B. M. (2006). Novel approach to the behavioural characterization of inbred mice: Automated home cage observations. Genes, Brain, \& Behavior, 5, 458-466. doi:10.1111/j.1601-183X.2005.00181.x

DunN, S. M. (1998). Software review: Optimas 6.5. Journal of ComputerAssisted Microscopy, 10, 53-54.

Galsworthy, M. J., Amrein, I., Kuptsov, P. A., Poletaeva, I. I., ZINN, P., RAU, A., ET AL. (2005). A comparison of wild-caught wood mice and bank voles in the Intellicage: Assessing exploration, daily activity patterns and place learning paradigms. Behavioural Brain Research, 157, 211-217. doi:10.1016/j.bbr.2004.06.021

GERLACH, G. (1996). Emigration mechanisms in feral house mice: A laboratory investigation of the influence of social structure, population density, and aggression. Behavioral Ecology \& Sociobiology, 39, 159-170. doi: $10.1007 / \mathrm{s} 002650050277$

Görtz, N., Lewejohann, L., Tomm, M., Ambrée, O., Keyvani, K., Paulus, W., \& SACHSER, N. (2008). Effects of environmental enrichment on exploration, anxiety, and memory in female TgCRND8 Alzheimer mice. Behavioural Brain Research, 191, 43-48. doi:10.1016/ j.bbr.2008.03.006

Janus, C., Pearson, J., Mclaurin, J., Mathews, P. M., Jiang, Y., SCHMidT, S. D., ET AL. (2000). $\mathrm{A}^{\beta}$ peptide immunization reduces behavioural impairment and plaques in a model of Alzheimer's disease. Nature, 408, 979-982. doi: $10.1038 / 35050110$

Kritzler, M., Lewejohann, L., Krüger, A., Raubal, M., \& SachSER, N. (2006). An RFID-based tracking system for laboratory mice in a semi-natural environment. Paper presented at the PERVASIVE 2006 Workshop-Pervasive Technology Applied Real-World Experiences With RFID and Sensor Networks, Dublin.

Lanari, A., Amenta, F., Silvestrelli, G., Tomassoni, D., \& ParNETTI, L. (2006). Neurotransmitter deficits in behavioural and psychological symptoms of Alzheimer's disease. Mechanisms of Ageing \& Development, 127, 158-165. doi:10.1016/j.mad.2005.09.016

Lewejohann, L., Reefmann, N., Widmann, P., Ambrée, O., Her- 
RING, A., KeYVANI, K., ET AL. (2009). Transgenic Alzheimer mice in a semi-naturalistic environment: More plaques, yet not compromised in daily life. Behavioural Brain Research, 201, 99-102. doi:10.1016/j .bbr.2009.01.037

Lewejohann, L., Skryabin, B. V., Sachser, N., Prehn, C., HeiduschKA, P., Thanos, S., ET AL. (2004). Role of a neuronal small nonmessenger RNA: Behavioural alterations in BC1 RNA-deleted mice. Behavioural Brain Research, 154, 273-289. doi:10.1016/j.bbr .2004 .02 .015

LLOYD, J. A. (1975). Social structure and reproduction in two freelygrowing populations of house mice (Mus musculus L.). Animal Behaviour, 23, 413-424. doi:10.1016/0003-3472(75)90089-5

Mackintosh, J. H. (1970). Territory formation by laboratory mice. Animal Behaviour, 18, 177-183.

Marshall, G. A., Fairbanks, L. A., Tekin, S., Vinters, H. V., \& CumMINGS, J. L. (2006). Neuropathologic correlates of activities of daily living in Alzheimer disease. Alzheimer Disease \& Associated Disorders, 20, 56-59. doi:10.1097/01.wad.0000201852.60330.16

Martin, P., \& Bateson, P. (1993). Measuring behaviour: An introductory guide (2nd ed.). Cambridge: Cambridge University Press.

Nelson, A. R., Johnson, C. L., Matter, W. J., \& Mannan, R. W. (2002). Tests of emigration in small mammals under experimental conditions. Canadian Journal of Zoology, 80, 2056-2060. doi:10.1139/z02-193
R Development Core Team (2008). R: A language and environment for statistical computing (Version 2.6.2). Vienna: R Foundation for Statistical Computing.

SAPOLSKY, R. M. (2005). The influence of social hierarchy on primate health. Science, 308, 648-652. doi:10.1126/science.1106477

Sebastiani, G., Krzywkowski, P., Dudal, S., Yu, M., Paquette, J., MaLO, D., ET AL. (2006). Mapping genetic modulators of amyloid plaque deposition in TgCRND8 transgenic mice. Human Molecular Genetics, 15, 2313-2323. doi:10.1093/hmg/dd1157

Touma, C., Ambrée, O., Görtz, N., Keyvani, K., Lewejohann, L., PALMe, R., ET AL. (2004). Age- and sex-dependent development of adrenocortical hyperactivity in a transgenic mouse model of Alzheimer's disease. Neurobiology of Aging, 25, 893-904. doi:10.1016/ j.neurobiolaging.2003.09.004

VON HoLST, D. (1998). The concept of stress and its relevance for animal behavior. In A. P. Møller, M. Milinski, \& P. J. B. Slater (Eds.), Advances in the study of behavior 27: Stress and behavior (pp. 1-131). San Diego: Academic Press.

(Manuscript received October 31, 2008; revision accepted for publication April 10, 2009.) 